Anno 34, 2019 / Fascicolo 1 / p. 154-156 - www.rivista-incontri.nl - http://doi.org/10.18352/incontri.10299 (C) The author(s) - Content is licensed under a Creative Commons Attribution 3.0 Unported License Publisher: Werkgroep Italië Studies, supported by Utrecht University Library Open Access Journals

\title{
L'eterna sfida per dominare il contemporaneo Il nuovo saggio di Carlo Tirinanzi De Medici
}

\author{
Recensione di: Carlo Tirinanzi De Medici, II romanzo italiano \\ contemporaneo. Dalla fine degli anni Settanta a oggi, Roma, \\ Carocci, 2018, 317 p., ISBN: 9788843088737, € 23,00.
}

\section{Carlo Baghetti}

Il contemporaneo è un mare agitato, attraversato da flutti e da flussi di temperature diverse che sono difficilmente distinguibili se non a distanza di tempo. Perciò il compito che si è posto Carlo Tirinanzi De Medici, come già se l'erano posto altri critici prima di lui - penso, tra gli altri, a Raffaele Donnarumma di Ipermodernità (2014), ad Alberto Casadei di Stile e tradizione nel romanzo contemporaneo (2007), a Daniele Giglioli di Senza trauma (2011), a Guido Mazzoni di Teoria del romanzo (2011) o, più di recente, a Gianluigi Simonetti de La letteratura circostante (2018), è difficile e rischioso. Tirinanzi De Medici si assume il rischio e cerca di gestirlo nella maniera più assennata possibile: suddivisione cronologica per decadi (o quasi: uno sguardo agli anni Settanta - il punto di partenza, poi 1979-1990, 1992-2000, 2001-2012, più uno scarno paragrafo finale intitolato 'Coda: tra presente e il museo', che analizza le ultimissime evoluzioni del panorama preso in considerazione); partizione del 'campo letterario' (p. 11) in correnti che oggi sembrano stabilizzate; analisi di testi e autori a cui la critica accorda rilevanza (più qualche importante "recupero"); trattazione sistematica delle problematiche di maggior rilievo che coinvolgono la narrativa contemporanea.

Il rischio maggiore corso nel saggio, a metà strada tra sistematizzazione storicocritica ed enunciazioni di teorie particolari su autori o gruppi di scrittori, è quello della semplificazione. In un volume che, nonostante l'ampiezza del periodo studiato e il grande numero di testi presi in considerazione (la ricca bibliografia è una bussola molto precisa per orientarsi nella contemporaneità letteraria italiana), resta agile, si ritrovano talvolta analisi eccessivamente nette, che tendono a condensare le problematiche, a omogeneizzare più che a distinguere le sfumature: è il prezzo da pagare al côté manualistico, che però non incide più di tanto sul valore generale del testo, che resta valido sia per studenti (di livello avanzato), i quali potranno farsi un'idea precisa della narrativa italiana contemporanea, sia per studiosi, i quali invece avranno la possibilità di confrontarsi con alcune interpretazioni originali del critico.

Tirinanzi De Medici parte dalla constatazione che l' anomalia' (p. 18, citando Asor Rosa) del caso italiano è quella di non aver avuto, come ad esempio in Francia, una koinè letteraria stabile e duratura. Concentrandosi sul dato linguistico il critico evoca lo scontro delle due koinè dominanti tra gli anni Cinquanta e Settanta, quella 'sperimentale' e quella del “"romanzo medio"', e sottolinea come solo entrando in crisi entrambe, tra anni Ottanta e Novanta, sia emersa una koinè letteraria italiana stabile, che negli anni Duemila porta a un 'primato definitivo della forma-romanzo' 
(p. 18). Primato raggiunto anche grazie all'implosione' (p. 22, citando stavolta Mazzoni), dopo sette secoli di dominio, del campo della poesia.

Tali sono le premesse per l'emersione del 'best seller all'italiana' (p. 25), cioè un 'romanzo medio "di qualità"' (ivi), categoria - secondo Tirinanzi De Medici - adatta a tenere insieme le opere di Eco, Calvino, Morante, le quali presentano una

ambientazione provinciale-borghese; [un] soggetto familiare, moderatamente corale; [un] piglio malinconico, più o meno rammemorante, che configura un romanzo intimista anche laddove si trattino i grandi eventi storici [...]; [una certa dose di] lirismo [...] - insomma quel 'romanticismo confezionato e razionalmente dosato [che] non contrappone al principio dell'aura un principio diverso, ma conserva l'aura, putrefatta, come alone fumogeno' dei prodotti midcult (ivi).

Nel leggere la produzione letteraria degli anni Ottanta e nel tentativo di spiegare l'esaurimento della componente sperimentale Tirinanzi De Medici evoca la caduta dell'a priori novecentesco (normalizzato da Adorno) che impediva agli scrittori di riprodurre il " "già detto"' (p. 31) (e il cui motto era make it new!). Questa è la chiave per comprendere il ritorno a un tipo di narrativa più piana e in cui una serie di dicotomie fondamentali (alto/basso, colto/popolare, fiction/non-fiction) sono venute meno.

A questo ritorno si lega il processo dell'affermazione progressiva dei generi letterari; dopo averne studiato la presenza in numerose opere pubblicate negli anni Ottanta, Tirinanzi De Medici giunge a chiedersi 'che forme assuma la narrativa italiana una volta immersa in un sistema in cui i generi hanno un ruolo centrale' (p. 86, corsivo mio). E a tal proposito il critico parla di 'tre diverse dominanti' (ivi), da una parte 'l'adesione a un ur-genere, con alterazioni all'interno delle regole generiche', come mostrano le analisi dei romanzi storici fatte nel paragrafo precedente; poi parla della 'commistione di generi letterari', e fa l'esempio del romanzo fantastorico o delle opere di Tabucchi che tendono a mescolare diversi modelli letterari; e infine 'la commistione tra generi discorsivi (giornalismo, ricerca, saggio, indagine sociologica ecc.)' (p. 87). Un'indicazione preziosa, quest'ultima, soprattutto alla luce della letteratura successiva, degli anni Duemila, per la quale Tirinanzi De Medici utilizza la definizione di narrativa 'a bassa finzionalità'.

All'interno del capitolo dedicato agli anni Ottanta la novità maggiore è rappresentata dalla duplice definizione che il critico propone per parlare dell'utilizzo dei codici extraletterari 'visti come chiavi alternative per accedere a strutture di senso che possano descrivere la realtà' (p. 96): romanzi a 'corto raggio' e a 'lungo raggio'. Nel primo gruppo Tirinanzi De Medici include quelle opere che limitano tale ricorso a pochi codici, come il televisivo o il cinematografico, e nelle quali si riscontra un 'afflato narrativo rivolto all'interiorità, secondo la lezione del novel' (ivi); al secondo gruppo, invece, appartengono opere che moltiplicano i suddetti codici, tentando d'includerne il maggior numero possibile (dove il paradigma è rappresentato da II pendolo di Foucault, definito 'romanzo-enciclopedia') e che appaiono 'dominate da un sistema narrativo improntato al romance' (ivi).

Nel terzo capitolo, dedicato agli anni Novanta, Tirinanzi De Medici rileva il 'desiderio di uno sguardo politico in senso lato' della narrativa (p. 113), messo in pratica attraverso strategie contrapposte: da una parte vi è la narrativa di genere, che procede verso l'iperfinzionalità; dall'altra, l'iperrealismo del documentario. Due tendenze 'apparentemente antitetiche' (p. 122, corsivo mio), ma che mostrano, nonostante remore e inquietudini, la fiducia nel 'mezzo narrativo e nelle sue capacità euristiche' (ivi). Seguono nel capitolo analisi approfondite sulla narrativa dei "cannibali", sulla narrativa di genere e in particolare quella "noir", sulla (poca) 
narrativa che s'ispira alla poetica postmodernista e, infine, sulla non-fiction, il territorio in cui l'intersezione di codici si fa più evidente.

Il quarto capitolo, che analizza la narrativa degli anni Duemila e fino al 2012, prende in considerazione - ancora una volta - il problema del 'ritorno alla realtà'. Tale tendenza, dice Tirinanzi De Medici, è sintomatica di un rinnovato impegno politico' (p. 185) da parte degli scrittori, per i quali si prospettano due vie: una prevede l'intervento diretto (Benedetti, Moresco, Scarpa, Voltolini), l'altra invece propone di conservare il tipico distacco letterario dagli eventi, per non banalizzare e appiattire la figura dello scrittore (Mozzi, Raimo). Il critico però mette in guardia il lettore da interpretazioni semplicistiche del ritorno al realismo: non si ripercorrono le 'direttrici novecentesche, sartriane' (p. 192) dell'impegno intellettuale, ma piuttosto, mettendo a frutto la lezione del postmodernismo letterario, il 'realismo viene trattato come uno tra i molti codici disponibili, da mischiare spesso ad altri' (ivi, corsivo mio).

A queste definizioni, ridefinizioni e precisazioni del campo letterario seguono una cinquantina di pagine in cui il critico mette a fuoco le tecniche e i topoi della koinè, gli utilizzi e gli scopi, e le varie forme che assume il realismo contemporaneo. Da tale capitolo emergono i pregi e i difetti del saggio di Tirinanzi De Medici: l'ampia sistematizzazione, supportata da una bibliografia molto ricca e varia, si basa su categorie critiche che risultano a volte leggermente permeabili. Altro aspetto poco convincente, particolarmente evidente in quest'ultimo capitolo, è l'alternanza tra la riflessione ad ampio spettro sulla narrativa e le analisi delle singole opere: sebbene sia una pratica frequente, ne II romanzo contemporaneo si registra una sproporzione tra questi due aspetti dell'argomentazione, con la presenza di veri e propri saggi su alcuni autori o opere (ad esempio su Celati, oppure su Gomorra, oppure i tre saggi che chiudono il quarto capitolo su Resistere non serve a niente, L'ubicazione del bene e Piove all'insù), che, per quanto precisi, solidi e talvolta necessari a riempire vuoti bibliografici, appaiono talvolta fuori contesto o eccessivi.

Nonostante i limiti ascrivibili a un'opera ambiziosa come quella di Tirinanzi De Medici, il saggio aggiunge un tassello non trascurabile al dibattito sulla contemporaneità. II romanzo italiano contemporaneo va posto quindi nello stesso scaffale dei testi citati all'inizio di questa recensione, che tentano di leggere, tematizzare e periodizzare gli ultimi anni di narrativa italiana.

\section{Carlo Baghetti}

Aix-Marseille Université

Centre Aixois d'Études Romanes

29, Avenue Robert Schuman

Aix-en-Provence (France)

carlo.baghetti@univ-amu.fr 